\title{
Behavioural Psychotherapy: Editorial Statement
}

Behavioural Psychotherapy is an international multidisciplinary journal for the publication of original research, of an experimental or clinical nature, that contributes to the theory, practice and evolution of behaviour therapy. As such, the scope of the journal is very broad, and articles relevant to most areas of human behaviour and human experience, which would be of interest to members of the helping and teaching professions, will be considered for publication.

As an applied science, the concepts, methodology and techniques of behavioural psychotherapy continue to change. The journal seeks both to reflect and to influence those changes.

While the emphasis is placed on empirical research, articles concerned with important theoretical and methodological issues as well as evaluative reviews of the behavioural literature are also published. In addition, given the emphasis of behaviour therapy on the experimental investigation of the single case, the Clinical Section of the journal publishes case studies using single case experimental designs. For the majority of designs this should include a baseline period with repeated measures; in all instances the nature of the quantitative data and the intervention must be clearly specified. Exceptionally, the journal will consider case studies where, although the interventions have not been experimentally evaluated, the treatment approach and/or prohlem dealt which is considered to be of particular importance and clear indicators of change are provided. Other types of case report can be submitted for the Brief Clinical Reports section (see below).

The following types of articles are suitable for Behavioural Psychotherapy:

1. Reports of original research employing experimental or correlational methods and using within or between subject designs.

2. Review or discussion articles which are based on empirical data and which have important new theoretical, conceptual or applied implications.

3. Brief reports and systematic investigations in single cases employing innovative techniques and/or approaches.

(C) 1992 British Association for Behavioural Psychotherapy 
Articles should concern original material which is neither published nor under consideration for publication elsewhere.

\section{Accelerated Publication Section}

In order to respond to rapid developments in the field, the journal includes an accelerated publication section. Articles accepted in this section will appear in the first possible issue of the journal, usually 3-6 months from receipt by the editors.

The accelerated publication section is intended to accommodate a small number of important papers. Such papers will include major new findings for which rapid dissemination would be of considerable benefit and impact. For example: reports of the results of important new clinical trials; innovative experimental results with major implications for theory or practice; other work of unusually high calibre.

Authors wishing to submit manuscripts for the accelerated publication section must briefly indicate in a covering letter (i) their reasons for requesting accelerated publication, (ii) that the material is original and has not been published or is not currently under consideration for publication elsewhere, (iii) that in the event of acceptance, the authors agree to the assignment of all copyrights to the manuscript in all forms and media to the British Association for Behavioural Psychotherapy. Authors should also indicate whether they wish the manuscript to be transferred to the normal (non-accelerated) review process if rejected for either of the stages of accelerated review outlined below. When submitting, the envelope should be clearly marked ACCELERATED REVIEW,

Accelerated review will proceed in two stages;

1. In the first stage, an editor and a reviewer will decide whether, in principle, the study merits accelerated publication. Authors will be informed within 14 days of receipt if this stage is not passed, and the manuscript will be withdrawn at that stage or continue in the normal (non-accelerated) review track, depending on the preference specified by the author(s).

2. Once passed in principle, the paper will then be subject to review by the same reviewers as in stage 1 using normal criteria. At the end of this stage, manuscripts will be either accepted (with or without minor alterations) or rejected for accelerated publication. Acceptance will depend on unanimity between the reviewer and editor. If accelerated publication is ruled out at this stage, a manuscript will revert to normal review if this option has been indicated by the author(s), otherwise it will simply be returned. 
In order to maximize the speed at which accelerated review proceeds, details of decisions on accelerated publication will be restricted to whether the manuscript has been accepted or not. Where applicable, minor alterations requested should be returned to the editors within three days of receipt. If a manuscript is transferred to the non-accelerated review track, the normal journal conventions regarding review decisions will be adhered to. The entire accelerated review process from receipt to final decisions should take an average of 2 weeks.

\section{Brief Clinical Reports}

Material suitable for this section includes unusual case reports, accounts of potentially important techniques, phenomena or observations; for example, descriptions of previously unreported techniques, outlines of available treatment manuals, descriptions of innovative variations of existing procedures, details of self-help or training packages, accounts of the application of existing techniques in novel settings and so on. The brief clinical reports section is intended to extend the scope of the clinical section. Submissions for this section should be no longer than five typescript, double spaced pages, and should include no more than six references. However, the author/s must also include with the submission copies an extended report which contains fuller clinical details. There are no restrictions on the size or format of this backup document. This may, for instance, be a treatment manual or a fully detailed case report, therapy transcript and so on. As both the report and the extended document will be sent for review, the usual number of copies are required. If a submission is accepted for publication as a Brief Clinical Report, the author/s must be prepared to send the fuller document to those requesting it, free of charge or at a price agreed with the editor to reflect the cost of materials involved. 